\title{
Red flag symptoms: detailed account of clinicopathological features in young-onset colorectal cancer
}

\author{
Ramish Riaz, Nosheen Masood, Arfa Benish \\ Department of Environmental Sciences (Biotechnology), Fatima Jinnah Women University, Rawalpindi, Pakistan
}

Background/Aims: Colorectal cancer has long been considered disease of the West, typically occurring in old age; however, the incidence is rising in Asia. The pattern of disease is quite different in Asia, occurring at a younger age and at an advanced stage. Recognition of disease at an early stage is still a challenge for physicians. Few data are available regarding young-onset colorectal cancer in Pakistan. We conducted this study to fill this gap and provide deeper insight into clinical symptoms and histopathological features of young-onset colorectal cancer. Methods: We collected data regarding clinical features by directly interviewing patients and obtaining histopathological data from hospital records. Patients aged less than 50 years were included in the study. Statistical analysis was performed using IBM SPSS version 20.0. Results: Results in 105 patients showed mean age at diagnosis was $35.90 \pm 9.39$, with male predominance; the majority of patients had no family history of colorectal cancer. Most patients had left-sided tumors with advance stage and intermediate grade (grade 2). Mucinous histology was common. Rectal bleeding was the first symptom for left-sided tumors, whereas most of the right-sided lesions presented with sudden obstruction. Conclusions: Painless rectal bleeding in the early thirties should alert physicians to advise appropriate investigation, as the majority of young-onset colorectal cancer patients develop painless bleeding 2 to 3 years before appearance of other symptoms. (Intest Res 2017;15:203-207)

Key Words: Colorectal neoplasms; Early-onset; Clinicopathological features

\section{INTRODUCTION}

Colorectal cancer (CRC) is the second most common cancer among male sex and the third most common among female sex worldwide. According to the International Agency for Research on Cancer, the highest incidence of CRC was reported in North America, Oceania, and Europe, especially in Eastern European countries. ${ }^{1}$ In the last few decades, 2to 3-fold increase in CRC incidence have been seen in East Asian countries. ${ }^{2}$ Pakistan lacks a nationwide cancer registry, and very little information is available regarding the incidence of CRC in Pakistan. However, a provincial level

Received July 29, 2016. Revised December 26, 2016.

Accepted December 27, 2016. Published online March 14, 2017

Correspondence to Ramish Riaz, Department of Environmental Sciences

(Biotechnology), Fatima Jinnah Women University, Mall Road, Rawalpindi,

Pakistan. Tel: +92-3335743898,E-mail: ramish_exclusive@hotmail.com

Financial support: None. Conflict of interest: None. registry in Punjab had shown that CRC was the third most common cancer among adults (>18 years) in $2015 .^{3}$

CRC has long been considered a disease of old age associated with westernization. Fifty years is considered an average age for CRC occurrence, and the incidence increases with age. Cancer occurring before this age is considered young-onset CRC, which accounts for $5 \%$ to $7 \%$ of all CRC cases. ${ }^{4}$ However, the trends of CRC in East Asian countries are quite different. Young-age CRC is common in these countries. ${ }^{5}$ Different studies conducted in Pakistan had also shown the average age of CRC to be 40 to 45 years. ${ }^{6,7}$

Young-onset CRC is strongly attributed to positive family history. ${ }^{8}$ Early diagnosis is a key to cure any disease. Screening programs in the West have played an important role in controlling CRC; however, screening mostly begins at age 50 to 60 years. ${ }^{9}$ The only way to control young-onset CRC is by paying attention to patients with suspicious symptoms. In Pakistan, records are generally unavailable regarding family

\footnotetext{
๑ Copyright 2017. Korean Association for the Study of Intestinal Diseases. All rights reserved.

This is an Open Access article distributed under the terms of the Creative Commons Attribution Non-Commercial License (http://creativecommons.org/licenses/by-nc/4.0)

which permits unrestricted non-commercial use, distribution, and reproduction in any medium, provided the original work is properly cited.
} 
history, clinical presentation, and histopathological details, so we conducted the current study to fill this gap.

\section{METHODS}

\section{Patients Population}

After obtaining informed consent from the patients and hospital authorities, data regarding clinical features were collected by directly interviewing the patients, and histopathological data were obtained from clinical records at different government hospitals in Islamabad and Rawalpindi from August 2014 to January 2016. A total of 155 patients were identified during the study period, of which 105 were less than 50 years of age. These patients were included in the study. Only patients who had histopathological disease confirmation and were staged according to TNM criteria were eligible.

\section{Statistical Analysis}

Data were analyzed by using IBM SPSS version 20.0. (IBM Corp., Armonk, NY, USA). Appropriate statistical tests were applied.

\section{RESULTS}

\section{Clinical Characteristics of Patients}

Most of the patients (83.2\%) in the study had a disease history of less than 1 year. Mean age for disease diagnosis

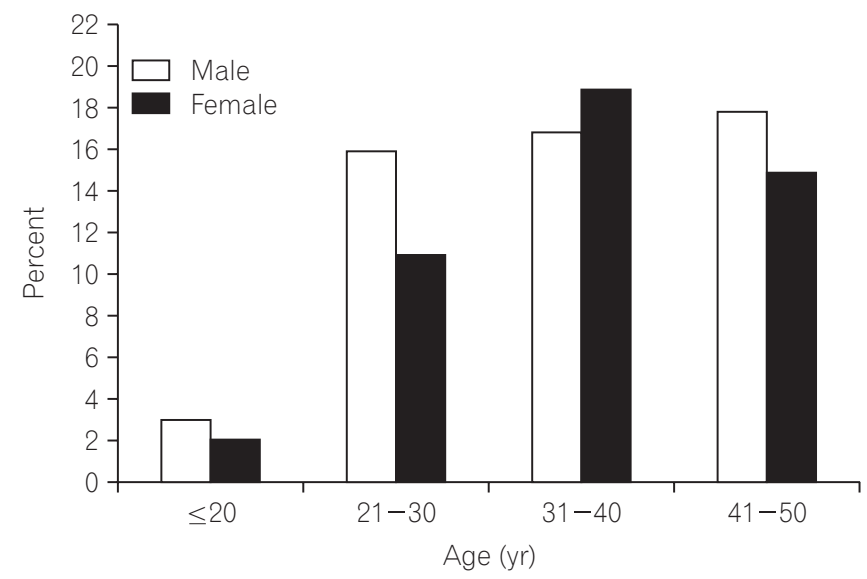

Fig. 1. Age versus sex distribution of colorectal cancer patients showing that the fourth age group at presentation, i.e., 41 to 50 years was the most common for male patients, whereas most female patients were aged between 31 and 40 years. was $35.90 \pm 9.39$. Most of the patients $(57 \%)$ were male. Fig. 1 shows age versus sex distribution of CRC patients. On histological grounds and according to Amsterdam and Bethesda criteria, no patient in this study had familial adenomatous polyposis or hereditary nonpolyposis CRC; all patients had sporadic CRC. No association was found between positive family history and young-onset CRC. Only $13 \%$ had a positive family history of disease; however, those with positive family history had more than one first-degree relative with CRC. Left-sided tumors were more common (81.7\%; chisquare, $40.18 ; P<0.001)$ than right-sided tumors. The most common site was the rectum, followed by the rectosigmoid junction and cecum (Fig. 2). Rectal bleeding was the most common initial symptom, followed by sudden obstruction

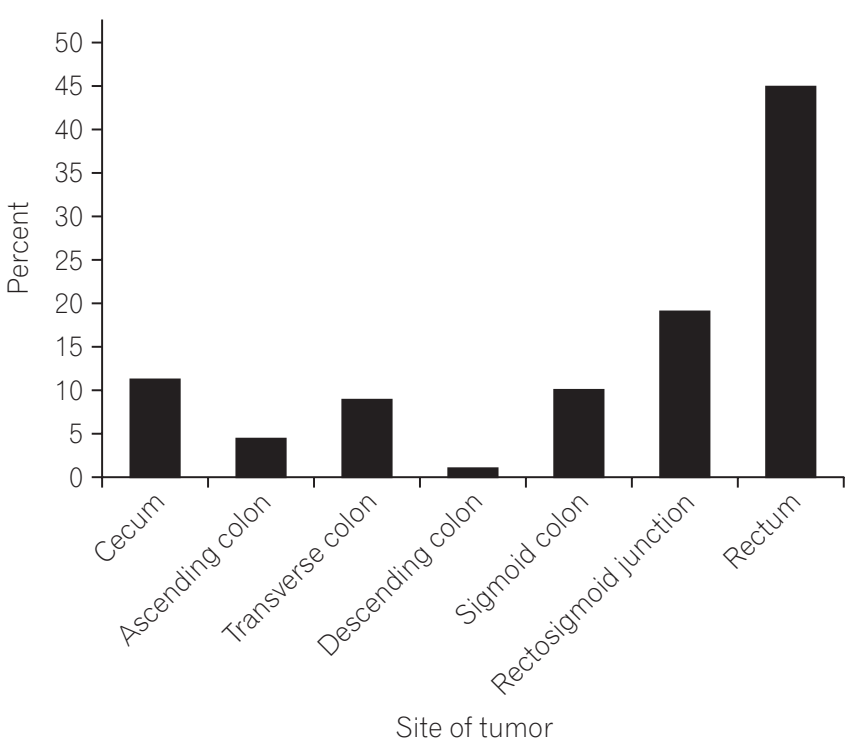

Fig. 2. Exact location of the tumor. The most common site was the rectum, followed by the rectosigmoid junction and the cecum.

Table 1. The First Symptom of Right-Sided versus Left-Sided Tumors

\begin{tabular}{lccr}
\hline \multicolumn{1}{c}{ Variable } & $\begin{array}{c}\text { Left-sided } \\
\text { tumor }\end{array}$ & $\begin{array}{c}\text { Right-sided } \\
\text { tumor }\end{array}$ & Total \\
\hline Initial symptom & & & \\
Chronic abdominal pain & $10(11.8)$ & $7(35.0)$ & $16.0 \%$ \\
Constipation & $9(10.5)$ & $1(5.0)$ & $9.6 \%$ \\
Blood in stool & $59(69.4)$ & 0 & $56.4 \%$ \\
$\begin{array}{l}\text { Sudden obstruction } \\
\text { (acute abdominal pain) }\end{array}$ & $6(7.0)$ & $12(60.0)$ & $17.0 \%$ \\
Diarrhea & $1(1.3)$ & 0 & $1.0 \%$ \\
Total & $85(100.0)$ & $20(100.0)$ & $105(100.0)$ \\
\hline
\end{tabular}

Values are presented as number (\%). Values are counted \% within right-sided versus left-sided tumors. 


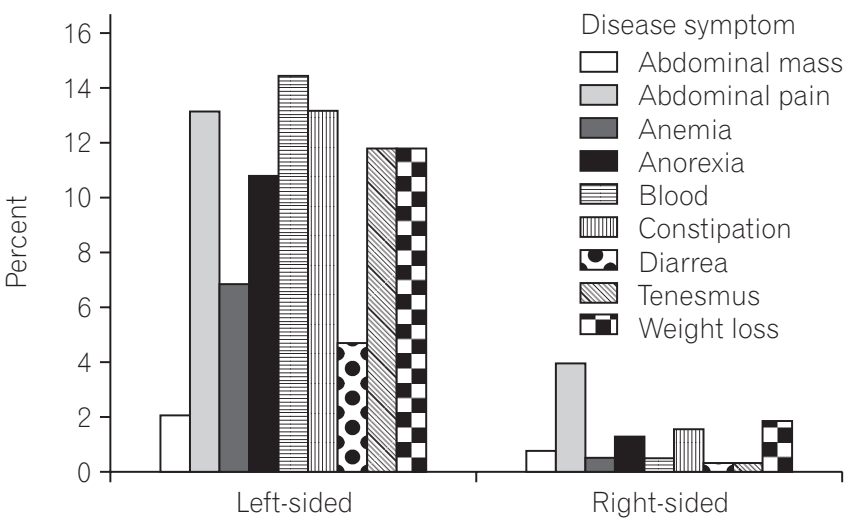

Fig. 3. Clinical presentation of right-sided versus left-sided tumors. Blood in stool was the most common symptom for left-sided masses, followed by constipation, tenesmus, and weight loss; abdominal pain was the most common clinical symptom for right-sided lesions, followed by weight loss.

and abdominal pain. However, when analyzed separately, most (69.1\%; chi-square, $47 ; P<0.0001)$ of the left-sided tumors presented with rectal bleeding, while most (60.0\%; chisquare, $41.04 ; P<0.001)$ of the right-sided tumors presented with sudden obstruction as their initial symptom (Table 1). Common symptoms for left-sided tumors were rectal bleeding, constipation, chronic abdominal pain, and tenesmus; however, for right-sided tumors, the most common initial symptom was acute abdominal pain, with sudden obstruction, followed by weight loss, constipation, and chronic abdominal pain. Fig. 3 shows the detailed analysis of presenting clinical symptoms in CRC patients in graphical format.

\section{Histologic Characteristics}

All of the tumors were adenocarcinomas. No other histological type was found. Among adenocarcinomas, most of the tumors (43.3\%) were either mucinous adenocarcinomas or non-mucinous, i.e., classic adenocarcinomas. Only 13.4\% were signet ring adenocarcinomas. No histological pattern differentiation was seen between right-sided and left-sided tumors. The most common histological grade (44.64\%) for left-sided masses was grade 2, i.e., moderately-differentiated adenocarcinoma, followed by well-differentiated tumors. The most common histological grade (40\%) for right-sided masses was grade 3, i.e., poorly-differentiated adenocarcinoma, followed by moderately-differentiated adenocarcinoma.

\section{TNM Staging}

Most of the tumors (40.6\%) were at TNM stage 4, followed
Table 2. TNM Staging of Colorectal Cancer Patients

\begin{tabular}{lc}
\hline \multicolumn{1}{c}{ Stage } & No. (\%) \\
\hline T1N0M0, stage 1 & $1(1.0)$ \\
T2N0M0, stage 1 & $7(6.6)$ \\
T3N0M0, stage 2A & $12(11.5)$ \\
T4N0M0, stage 2B & $5(4.7)$ \\
T1 or T2, N1M0, stage 3A & $1(1.0)$ \\
T1 or T2, N2M0, stage 3A & $3(3.0)$ \\
T3N1M0, stage 3B & $16(15.2)$ \\
T3N2M0, stage 3C & $9(8.5)$ \\
T4N1M0, stage 3C & $6(5.5)$ \\
T4N2M0, stage 3C & $3(3.0)$ \\
Any T with M1, stage 4 & $42(40.0)$ \\
Total & $105(100.0)$ \\
\hline
\end{tabular}

by stage $3(36.9 \%)$. Advanced stages were common among both sexes. Negative correlation (-0.206) was found between age and stage of disease, i.e., the younger the age, the more likely for a tumor to be invasive. Tumors presenting with grade 3 histology were more likely (OR, 5.77; RR, 1.5; $P=0.02$ ) to be advanced, rather than grade 1 tumors. Most common site (57.14) for metastasis was liver, followed by peritoneum and lungs. Table 2 shows the detailed TNM staging.

\section{DISCUSSION}

In Pakistan, few studies have been conducted on CRC. Studies have mostly focused on representing different cancers in different age groups. Such studies identified the increase in CRC incidence in a younger population in Pakistan. The current study was conducted to highlight clinicopathological features of young-onset CRC in Pakistan, so that appropriate steps could be taken to diagnose and cure CRC at an early stage.

The main results of the current study show that most young-onset CRC patients had a negative family history, leftsided masses, and advanced stage, and were symptomatic at the time of presentation. Right-sided tumors are traditionally associated with female sex. ${ }^{10}$ However, in our study, no association (OR, 1.09; RR, 1.07; $P=0.86$ ) was found between sex and type of cancer. Worldwide, positive family history has been associated with young-onset CRC; however, our results differed. ${ }^{11}$

Most patients with left-sided tumors presented with rectal bleeding as their initial symptom, while those with rightsided tumors presented with sudden bowel obstruction. 
Symptom presentation in CRC patients in this study was consistent with that reported by other studies; regardless of age. ${ }^{12}$ Most patients with left-sided tumors developed rectal bleeding 1 to 2 years prior to other symptoms. Early colonoscopy in young patients with rectal bleeding could help in early diagnosis.

Mucinous and signet ring type adenocarcinomas are considered less common than classic adenocarcinomas. ${ }^{13} \mathrm{In}$ the West, where old age-onset CRC is common, only $10 \%$ to $20 \%$ of all CRCs are mucinous. ${ }^{14}$ However in our study, most CRCs were mucinous and classic adenocarcinomas, while signet ring cell carcinomas were rare. A high rate of mucinous carcinomas in the present study could be linked with younger age, as different studies have shown that mucinous adenocarcinomas are common in younger age groups and are associated with late diagnosis, advanced stage, and poor prognosis. ${ }^{13}$ Our results were in agreement, as the stage of mucinous lesions was more advanced than that of classic adenocarcinoma in the current study; however, the grade of classic adenocarcinomas showed the opposite result.

Diagnosis at an early stage of cancer is the key to good prognosis. Usually, CRC is diagnosed at early stages, at a mean age of 70 years. ${ }^{15}$ A study by Ahnen et al. ${ }^{16}$ observed that young-onset CRCs are mostly diagnosed at advanced stages and have a poor prognosis, which is in agreement with the findings in the present study. In another study by Amini et al. ${ }^{17}$ conducted in Pakistan, a negative correlation was found between CRC and age. Poorly-differentiated adenocarcinomas are a hallmark of advanced stage and poor prognosis; our study showed the same findings. ${ }^{18}$ CRC spreads mostly to the liver followed by peritoneum and lungs. Our findings were the same.

A cross-sectional study by Zahir et al. ${ }^{19}$ also reported a higher prevalence of CRC among young patients in 2004 to 2011. According to their study, 581 patients were reported, among whom $32 \%$ were younger than 45 years. In contrast, the present study duration was shorter than that of the above mentioned study (2014 to 2016), and early onset of CRC was present in $67.7 \%$ of patients. This indicates a higher prevalence of CRC among younger individuals. Our results were in agreement with a study by Amini et al., ${ }^{17}$ which reported a higher incidence of CRC in patients aged less than 40 years; however, as highlighted by the authors, their study population was small.

This study was conducted to highlight the clinicopathological features of CRC in young age groups so that appropriate steps can be taken for early diagnosis. Our study has certain limitations. A comparison between young-onset and late- onset CRC was not discussed because of the small number of late-onset patients reported during the study period. However, this indicates that the incidence of CRC among young individuals is increasing. Even though young-onset CRC is not asymptomatic, patients often ignore the symptoms. This might result in late diagnosis of the disease. Most physicians consider rectal bleeding as a result of hemorrhoids or fissures and prescribe mild laxatives and local anesthetics. Therefore, rectal bleeding at a young age should raise suspicion, and appropriate investigation must be advised. This can help in diagnosis of CRC at early stages, and early treatment can lead to increased survival rate, avoiding a poor cancer prognosis. However, the relationship between youngonset and advanced stage presentation cannot be ruled out because most patients included in the study had a history of disease for less than 1 year.

The pattern of CRC in Asia is quite different from that in the West. This study was conducted to provide insight into the clinicopathological features of CRC in Pakistan. Most patients developed cancer at young age and had advanced disease and rare histological types, with no family history; this creates a challenge for diagnosis at an early stage and cure. In other countries, a positive family history plays an important role in young-onset CRC, which helps in early diagnosis of a population at risk. However, we did not find any association between family history and CRC. This study was conducted to show the early features of CRC, which can help in early diagnosis. Rectal bleeding was the most common symptom among patients with left-sided masses, and was present 1 to 2 years before appearance of any other symptom. There is a need to pay attention to these symptoms, and appropriate investigation must be advised, so that CRC can be diagnosed at an early stage and managed successfully. There is also a need for genetic analysis in these patients, which may help in finding a culprit gene in young-onset CRC. Studies comparing young-onset CRC features with those in late-onset patients are needed.

\section{ACKNOWLEDGEMENTS}

Very special thanks to Saima Shakeel Malik and Riffat Shabbir who helped us a lot during the whole project. Special thanks to all the patients who willingly contributed in this study. 


\section{REFERENCES}

1. Center MM, Jemal A, Smith RA, Ward E. Worldwide variations in colorectal cancer. CA Cancer J Clin 2009;59:366-378.

2. Pourhoseingholi MA. Epidemiology and burden of colorectal cancer in Asia-Pacific region: what shall we do now? Transl Gastrointest Cancer 2014;3:169-173.

3. Punjab Cancer Registry. Punjab cancer registry report-4. Lahore: Punjab Cancer Registry, 2016.

4. Ballester V, Rashtak S, Boardman L. Clinical and molecular features of young-onset colorectal cancer. World J Gastroenterol 2016;22:1736-1744.

5. Deen KI, Silva H, Deen R, Chandrasinghe PC. Colorectal cancer in the young, many questions, few answers. World J Gastrointest Oncol 2016;8:481-488.

6. Ahmed A, Afzal A, Asif HM, et al. Changing trends of presentation in colorectal carcinoma. Pak J Med Sci 2014;8:233-237.

7. Hadi NI, Kafil N, Waseem B, Alamgir M. Incidence of colorectal carcinoma: is there a "shift to the right"? Pak J Pharmacol 2009;26:1-5.

8. Rosato V, Bosetti C, Levi F, et al. Risk factors for young-onset colorectal cancer. Cancer Causes Control 2013;24:335-341.

9. Wilson JA. Colon cancer screening in the elderly: when do we stop? Trans Am Clin Climatol Assoc 2010;121:94-103.

10. Jess P, Hansen IO, Gamborg M, Jess T; Danish Colorectal Cancer Group. A nationwide Danish cohort study challenging the categorisation into right-sided and left-sided colon cancer. BMJ Open 2013;3:e002608. doi: 10.1136/bmjopen-2013-002608.

11. Baichoo E, Boardman LA. Genetics of young onset colorectal cancer. Hereditary Genet 2013;3:124.
12. Dozois EJ, Boardman LA, Suwanthanma W, et al. Young-onset colorectal cancer in patients with no known genetic predisposition: can we increase early recognition and improve outcome? Medicine (Baltimore) 2008;87:259-263.

13. Nitsche U, Zimmermann A, Späth C, et al. Mucinous and signetring cell colorectal cancers differ from classical adenocarcinomas in tumor biology and prognosis. Ann Surg 2013;258:775782.

14. Benedix F, Kuester D, Meyer F, Lippert H. Influence of mucinous and signet-ring cell differentiation on epidemiological, histological, molecular biological features, and outcome in patients with colorectal carcinoma. Zentralbl Chir 2013;138:427-433.

15. McPhail S, Johnson S, Greenberg D, Peake M, Rous B. Stage at diagnosis and early mortality from cancer in England. Br J Cancer 2015;112 Suppl 1:S108-S115.

16. Ahnen DJ, Wade SW, Jones WF, et al. The increasing incidence of young-onset colorectal cancer: a call to action. Mayo Clin Proc 2014;89:216-224.

17. Amini AQ, Samo KA, Memon AS. Colorectal cancer in younger population: our experience. J Pak Med Assoc 2013;63:12751277.

18. Barresi V, Reggiani Bonetti L, Ieni A, Caruso RA, Tuccari G. Histological grading in colorectal cancer: new insights and perspectives. Histol Histopathol 2015;30:1059-1067.

19. Zahir MN, Azhar EM, Rafiq S, Ghias K, Shabbir-Moosajee M. Clinical features and outcome of sporadic colorectal carcinoma in young patients: a cross-sectional analysis from a developing country. ISRN Oncol 2014;2014:461570. 